\title{
Production of heavy particle pairs via photon-photon processes at the LHC in proton-proton scattering
}

\author{
Marta Luszczak* \\ College of Natural Sciences, Institute of Physics, University of Rzeszów, \\ ul. Pigonia 1, PL-35310 Rzeszów, Poland
}

E-mail: luszczak@ur .edu.pl

We discuss the production of $W^{+} W^{-}$pairs and $t \bar{t}$ quark-antiquark pairs in proton-proton collisions induced by two-photon fusion including the transverse momenta of incoming photons. We focus on processes with single and double proton dissociation. Highly excited remnant systems hadronise producing particles that can be vetoed in the calorimeter. We calculate associated gap survival factors. The gap survival factors depend on the process, mass of the remnant system and collision energy. The rapidity gap survival factor due to remnant fragmentation for double dissociative (DD) collisions is smaller than that for single dissociative (SD) process. We observe approximate factorisation: $S_{R, D D} \approx S_{R, S D}^{2}$ when imposing rapidity veto. For the $W^{+} W^{-}$final state, the remnant fragmentation leads to a taming of the cross section when the rapidity gap requirement is imposed. Also for $t \bar{t}$ quark-antiquark pairs such a condition reverses the hierarchy observed for the case when such condition is taken into account.

40th International Conference on High Energy physics - ICHEP2020

July 28 - August 6, 2020

Prague, Czech Republic (virtual meeting)

\footnotetext{
${ }^{*}$ Speaker
} 


\section{Introduction}

Photon-induced processes in proton-proton interactions have become very topical recently. Experimentally they can be separated from other competing processes by imposing rapidity gaps around the electroweak vertex. Both charged lepton pairs $l^{+} l^{-}[1-5]$ and electroweak gauge bosons $W^{+} W^{-}[6,7]$ were recently studied experimentally at the Large Hadron Collider. In particular, processes with $W^{+} W^{-}$are of special interest in the context of searches beyond Standard Model $[8,9]$. There are, in general, different categories of such processes depending on whether the proton stays intact or undergoes an electromagnetic dissociation (see e.g. [10,11]).

The $W^{+} W^{-}$production in proton-proton processes via the $\gamma \gamma \rightarrow W^{+} W^{-}$subprocess was studied in collinear [12] and transverse momentum dependent factorisation [13] approaches. In our paper [13] we showed that rather large photon virtualities and large mass proton excitation are characteristic for the $\gamma \gamma \rightarrow W^{+} W^{-}$induced processes. Our main aim was to estimate gap survival factor associated with the remnant hadronisation, which destroys the rapidity gap. In [14] we concentrated on the effect related to remnant fragmentation and its destoying of the rapidity gap. Finally in [15] we calculated the cross section for the photon-photon contribution for the $p p \rightarrow t \bar{t}$ reaction including also effects of gap survival probability.

\section{Results for $W^{+} W^{-}$pair production}

In Fig. 1 we show the two-dimensional distributions in pseudorapidity of particles from proton $X\left(\eta_{X}^{\mathrm{ch}}\right)$ and proton $Y\left(\eta_{Y}^{\mathrm{ch}}\right)$ for different ranges of masses of the centrally produced system. For illustration the region relevant for ATLAS and CMS pseudorapidity coverage is pictured by the thin dashed square.

The two dimensional plots are not sufficient to see the dependence of the associated gap survival factor on the mass of the centrally produced system.

We quantify this effect, see Table 1 , by showing average remnant rapidity gap factors for different ranges of $M_{W W}$ masses. There we observe a rather mild dependence. The remnant rapidity gap survival factor at fixed $\eta_{\text {cut }}$ becomes larger at higher collision energies. 


\begin{tabular}{|c|c|c|c|c|c|c|}
\hline \multirow{2}{*}{ Contribution } & \multicolumn{2}{|c|}{$S_{R, S D}\left(\left|\eta^{\mathrm{ch}}\right|<2.5\right)$} & \multicolumn{2}{c|}{$\left(S_{R, S D}\right)^{2}\left(\left|\eta^{\mathrm{ch}}\right|<2.5\right)$} & \multicolumn{2}{c|}{$S_{R, D D}\left(\left|\eta^{\mathrm{ch}}\right|<2.5\right)$} \\
\cline { 2 - 7 } & $8 \mathrm{TeV}$ & $13 \mathrm{TeV}$ & $8 \mathrm{TeV}$ & $13 \mathrm{TeV}$ & $8 \mathrm{TeV}$ & $13 \mathrm{TeV}$ \\
\hline$\left(2 M_{W W}, 200 \mathrm{GeV}\right)$ & $0.763(2)$ & $0.769(2)$ & $0.582(4)$ & $0.591(4)$ & $0.586(1)$ & $0.601(2)$ \\
\hline$(200,500 \mathrm{GeV})$ & $0.787(1)$ & $0.799(1)$ & $0.619(2)$ & $0.638(2)$ & $0.629(1)$ & $0.649(1)$ \\
\hline$(500,1000 \mathrm{GeV})$ & $0.812(2)$ & $0.831(2)$ & $0.659(3)$ & $0.691(3)$ & $0.673(2)$ & $0.705(2)$ \\
\hline$(1000,2000 \mathrm{GeV})$ & $0.838(7)$ & $0.873(5)$ & $0.702(12)$ & $0.762(8)$ & $0.697(5)$ & $0.763(6)$ \\
\hline full range & $0.782(1)$ & $0.799(1)$ & $0.611(2)$ & $0.638(2)$ & $0.617(1)$ & $0.646(1)$ \\
\hline
\end{tabular}

Table 1: Average rapidity gap survival factor related to remnant fragmentation for single dissociative and double dissociative contributions for different ranges of $M_{W W}$. All uncertainties are statistical only.

\begin{tabular}{|c|c|c|}
\hline Contribution & No cuts & $y_{\text {jet }}$ cut \\
\hline $\begin{array}{c}\text { elastic-elastic } \\
\text { elastic-inelastic }\end{array}$ & 0.292 & 0.292 \\
inelastic-elastic & 0.544 & 0.439 \\
inelastic-inelastic & 0.983 & 0.622 \\
\hline all contributions & 2.36 & 1.79 \\
\hline
\end{tabular}

Table 2: Cross section in fb at $\sqrt{s}=13 \mathrm{TeV}$ for different components (left column) and the same when the extra condition on the outgoing jet $\left|y_{\text {jet }}\right|>2.5$ is imposed.

In Fig. 2 we show the distribution in $\eta_{\text {cut }}$ for the double dissociation process. We predict a strong dependence on $\eta_{\text {cut }}$. It would be valuable to perform experimental measurements with different $\eta_{\text {cut }}$.

\section{Results for $t \bar{t}$ pairs production}

The summed inclusive cross section at $\sqrt{s}=13 \mathrm{TeV}$ is $2.36 \mathrm{fb}$. This is a rather small number in comparison with other inclusive production mechanisms. In the right panel of Table 2 we show results when a rapidity gap ${ }^{1}$ in the central region, for $-2.5<y<2.5$ is required in addition. In principle, imposing this condition requires modelling of the full final state, as we did for the case of $W^{+} W^{-}$production. As in each event we have the full four-momentum of the virtual photon(s), as well as the invariant masses of the proton remnants, the four-momenta of the recoiling jet(s) can be reconstructed. To a good accuracy the rapidity gap condition is equivalent to requiring that the recoiling jets fulfill $\left|y_{\text {jet }}\right|>2.5$.

The same is true for the distribution in $t \bar{t}$ invariant mass (see the left panel of Fig.3). The distributions are almost identical and differ only by normalisation. In the right panel of Fig. 3 we show similar results when conditions on outgoing light quark/antiquark jets are imposed. The extra condition leads to a lowering of the cross section with only very small modification of the shape of the $M_{t \bar{t}}$ distribution.

In Fig.4 we show distributions in $M_{X}$ for a fixed $M_{Y}$ (left panel) and in $M_{Y}$ for a fixed $M_{X}$ (right panel). The distributions are arbitrarily normalized to the same integral. All the distributions

\footnotetext{
${ }^{1}$ That means that no additional particle is produced except $t$ or $\bar{t}$
} 

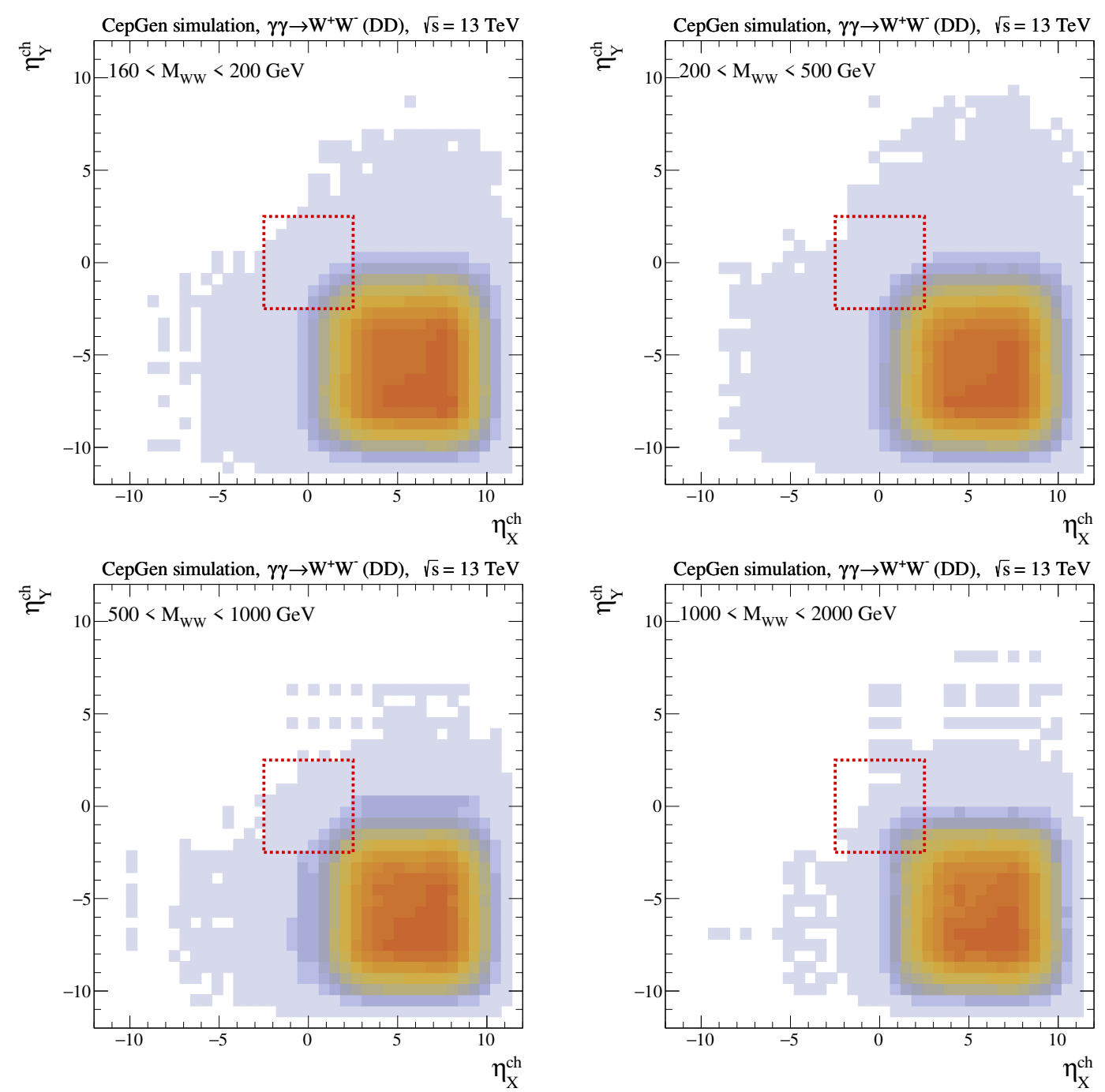

Figure 1: Two-dimensional $\left(\eta_{X}^{\mathrm{ch}}, \eta_{Y}^{\mathrm{ch}}\right)$ distribution for four different windows of $M_{W W}$ : $\left(2 M_{W}, 200 \mathrm{GeV}\right)$, $(200,500 \mathrm{GeV}),(500,1000 \mathrm{GeV}),(1000,2000 \mathrm{GeV})$. The square shows pseudorapidity coverage of ATLAS or CMS inner tracker.

coincide.

\section{Conclusions}

In this presentation we have discussed the quantity called remnant gap survival factor for the $p p \rightarrow W^{+} W^{-}$and $p p \rightarrow t \bar{t}$ reactions initiated via photon-photon fusion. We use our formalism developed for the inclusive case [13] which includes transverse momenta of incoming photons. The partonic formalism has been supplemented by including remnant fragmentation that can spoil the rapidity gap usually used to select the subprocess of interest. We quantify this effect by defining the remnant gap survival factor which in general depends on the reaction, kinematic variables and details of the experimental set-ups. 

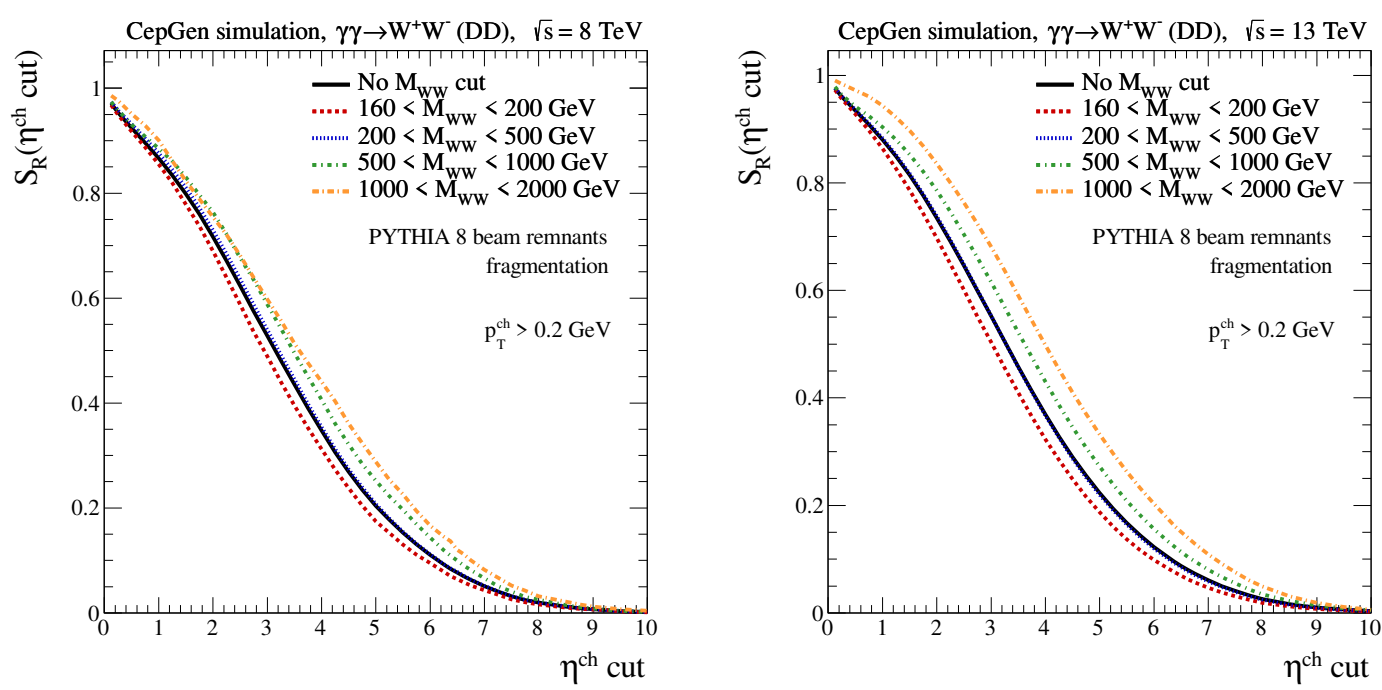

Figure 2: Gap survival factor for double dissociation as a function of the size of the pseudorapidity veto applied on charged particles emitted from proton remnants, for the diboson mass bins defined in the text and in the figures for $\sqrt{s}=8 \mathrm{TeV}$ (left) and $13 \mathrm{TeV}$ (right).
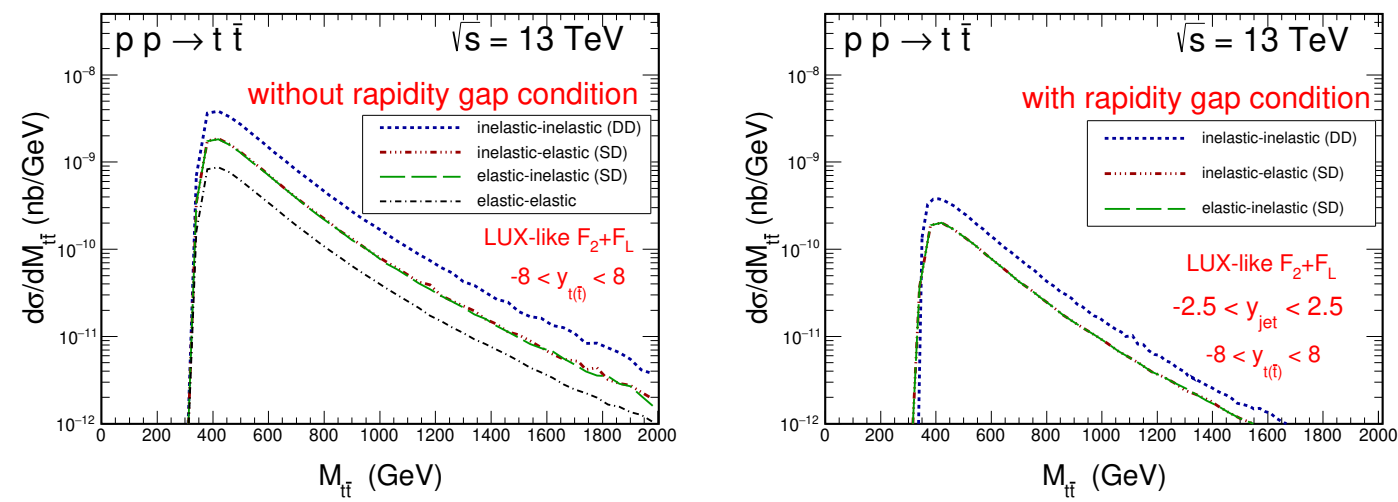

Figure 3: $t \bar{t}$ invariant mass distribution for different components defined in the figure. The left panel is without imposing the condition on the struck quark/antiquark and the right panel includes the condition.

The cross sections for production of $t \bar{t}$ pairs via $\gamma^{*} \gamma^{*}$ fusion summed over the different categories of processes is about $2.36 \mathrm{fb}$ (full phase space), i.e. rather small compared to the standard inclusive $t \bar{t}$ cross section (of the order of nb). Our results imply that for the production of such heavy objects as $t$ quark and $\bar{t}$ antiquark the virtuality of the photons attached to the dissociative system are very large $\left(Q^{2}<10^{4} \mathrm{GeV}^{2}\right)$.

\section{References}

[1] S. Chatrchyan et al. [CMS Collaboration], JHEP 1201 (2012) 052 [arXiv:1111.5536 [hepex]]. 

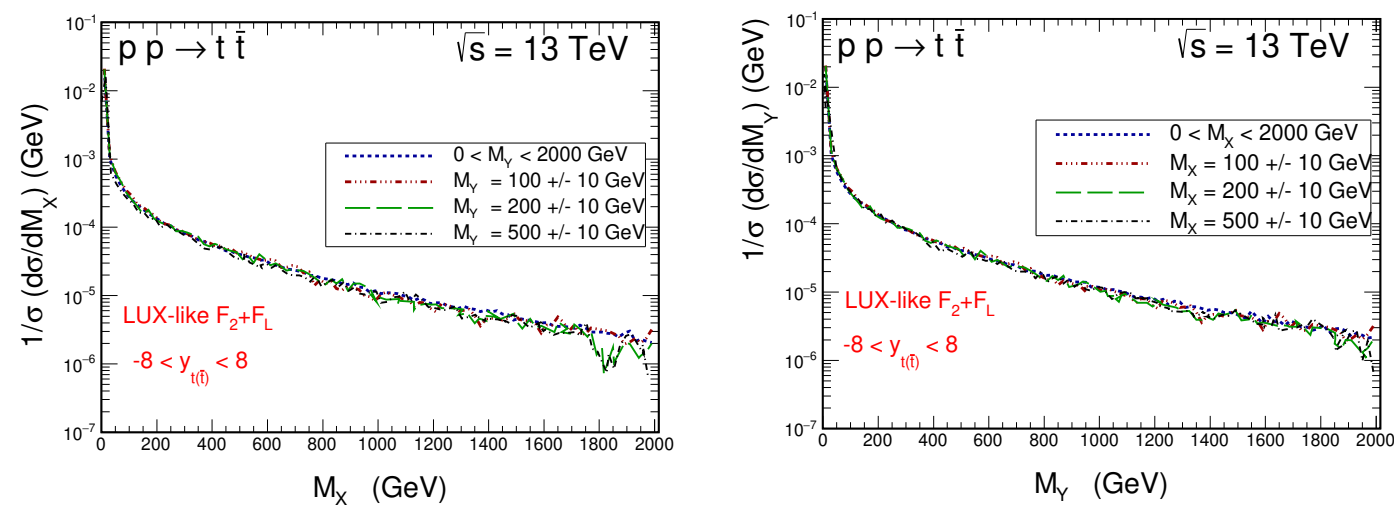

Figure 4: Distribution in $M_{X}$ for different windows of $M_{Y}$ (left) and as a function of $M_{Y}$ for different windows of $M_{X}$ (right).

[2] S. Chatrchyan et al. [CMS Collaboration], JHEP 1211 (2012) 080 [arXiv:1209.1666 [hepex]].

[3] G. Aad et al. [ATLAS Collaboration], Phys. Lett. B 749 (2015) 242 [arXiv:1506.07098 [hep-ex]].

[4] A. M. Sirunyan et al. [CMS and TOTEM Collaborations], JHEP 1807 (2018) 153 [arXiv:1803.04496 [hep-ex]].

[5] M. Aaboud et al. [ATLAS Collaboration], Phys. Lett. B 777 (2018) 303 [arXiv:1708.04053 [hep-ex]].

[6] V. Khachatryan et al. [CMS Collaboration], JHEP 1608, 119 (2016) [arXiv:1604.04464 [hep-ex]].

[7] M. Aaboud et al. [ATLAS Collaboration], Phys. Rev. D 94, no. 3, 032011 (2016) [arXiv:1607.03745 [hep-ex]].

[8] E. Chapon, C. Royon and O. Kepka, Phys. Rev. D 81 (2010) 074003 [arXiv:0912.5161 [hep-ph]].

[9] T. Pierzchala and K. Piotrzkowski, Nucl. Phys. Proc. Suppl. 179-180, 257 (2008) [arXiv:0807.1121 [hep-ph]].

[10] G. G. da Silveira, L. Forthomme, K. Piotrzkowski, W. Schäfer and A. Szczurek, JHEP 1502 (2015) 159 [arXiv:1409.1541 [hep-ph]].

[11] M. Luszczak, W. Schäfer and A. Szczurek, Phys. Rev. D 93, no. 7, 074018 (2016) [arXiv:1510.00294 [hep-ph]].

[12] M. Luszczak, A. Szczurek and C. Royon, JHEP 1502 (2015) 098 [arXiv:1409.1803 [hep-ph]]. 
[13] M. Luszczak, W. Schäfer and A. Szczurek, JHEP 1805 (2018) 064 [arXiv:1802.03244 [hep$\mathrm{ph}]]$.

[14] L. Forthomme, M. Luszczak, W. Schäfer and A. Szczurek, Phys. Lett. B 789 (2019) 300 [arXiv:1805.07124 [hep-ph]].

[15] M. Luszczak, L. Forthomme, W. Schäfer and A. Szczurek, JHEP 1902 (2019) 100 [arXiv:1810.12432 [hep-ph]]. 